University of Nebraska - Lincoln

DigitalCommons@University of Nebraska - Lincoln

2009

\title{
A location analysis approach for military maintenance scheduling with geographically dispersed service areas
}

Dale L. Overholts II

Air Force Institute of Technology

John E. Bell

Air Force Institute of Technology

Marvin A. Arostegui

Air Force Institute of Technology

Follow this and additional works at: https://digitalcommons.unl.edu/usafresearch

Part of the Aerospace Engineering Commons

Overholts II, Dale L.; Bell, John E.; and Arostegui, Marvin A., "A location analysis approach for military maintenance scheduling with geographically dispersed service areas" (2009). U.S. Air Force Research. 27. https://digitalcommons.unl.edu/usafresearch/27

This Article is brought to you for free and open access by the U.S. Department of Defense at DigitalCommons@University of Nebraska - Lincoln. It has been accepted for inclusion in U.S. Air Force Research by an authorized administrator of DigitalCommons@University of Nebraska - Lincoln. 


\title{
A location analysis approach for military maintenance scheduling with geographically dispersed service areas ${ }^{2}$
}

\author{
Dale L. Overholts II, John E. Bell*, Marvin A. Arostegui \\ Department of Operational Sciences, Air Force Institute of Technology, 2950 Hobson Way, Wright Patterson Air Force Base, OH 45433, USA
}

Received 26 January 2008; accepted 19 May 2008

Available online 24 May 2008

\begin{abstract}
This research uses a two-stage maximal covering location problem (MCLP) model to develop Inter continental ballistic missile (ICBM) maintenance schedules for the US Air Force. Solutions are compared to actual missile maintenance activities accomplished at F. E. Warren Air Force Base (AFB), Wyoming in May 2005. Sensitivity analysis is performed to determine the impact of altering security response times and the number of security patrol areas on the quality of daily maintenance schedules and personnel usage. Results indicate marked improvement over traditional Air Force scheduling methods. In addition sensitivity analysis identifies the levels at which the quality and quantity of maintenance performance is impacted.

Published by Elsevier Ltd.
\end{abstract}

Keywords: Decision making/process; Scheduling; Location; Maintenance; Optimization

\section{Introduction}

Since the early 1960s, Intercontinental Ballistic Missile's (ICBMs) have served as the long-range attack segment of the US nuclear deterrent force. The Minuteman III weapon system is the only remaining US Air Force ICBM system. The Air Force's 500 Minuteman III missiles are dispersed among three Air Force bases: 200 missiles at Malmstrom AFB, Montana; 150 missiles at Minot AFB, North Dakota; and 150 missiles at F. E. Warren AFB, Wyoming. ICBMs are stored in unmanned, hardened, underground structures known as launch facilities. A separate missile alert facility serves as the center of assigned security patrol areas,

\footnotetext{
This manuscript was processed by Area Editor B. Lev.

* Corresponding author. Tel.: +14789262017, fax: +14789265912 .

E-mail address: john.bell@ecats.gcsu.edu (J.E. Bell).
}

the staging point for security forces teams deployed to the missile complex, and as an area away from the main base where maintenance personnel can remain overnight. One missile alert facility controls 10 launch facilities and makes up a flight; five flights make up a squadron. F. E. Warren Air force base (AFB) which is the focus for this study, has three Minuteman III missile squadrons, each responsible for 50 ICBMs.

An ordered priority system, from 1 to 9 , is used to dictate precedence for maintenance activities performed for ICBMs [1]. Priority 1 maintenance activities are the most crucial, often requiring immediate repair or replacement of critical equipment to maintain the weapon system, while priority 9 activities are generally minor repairs that have no impact on the missile operation. This priority system determines which launch facilities will be scheduled for maintenance, given a constrained number of maintenance teams and security personnel. If a maintenance team requires direct access to the 
missile or its classified components, the team must be accompanied by a security escort team (SET), which protects the launch facility while maintenance is being accomplished. Additional security teams monitor activity within a designated security patrol area and stand ready to respond to potentially hostile activities within the patrol area.

Maintenance of the Minuteman III weapon system requires maintenance and security teams travel to one or more of 150 launch facilities, all of which are geographically isolated from major population centers. Travel time from F. E. Warren AFB, the main support base, to the launch facilities can range from less than $25 \mathrm{~min}$ to over $2.5 \mathrm{~h}$. Teams are limited to a $16-\mathrm{h}$ timeline, which begins as soon as a team arrives at the F. E. Warren AFB work center to make travel preparations, and ends after all maintenance actions are completed, or once the team arrives at one of the 15 missile alert facilities dispersed throughout the 12,600 square-mile missile complex. Each missile alert facility serves as the hub of one security patrol area, allowing for up to 15 total security patrol areas. At the time of this research, the available missile security personnel could support two security patrol areas per day, which had a maximum coverage radius of 60-min travel time from the missile alert facility. Missile alert facilities have already been built, so their locations are fixed. However, the supported security patrol areas can shift between missile alert facilities daily, depending on which area(s) of the missile complex the launch facility maintenance activities are needed. All launch facility maintenance activities that require access to the missile must fall within at least one security patrol area, or maintenance cannot be performed. As such, missile maintenance schedulers must try to geographically cluster maintenance activities within these two security patrol areas to forego maintenance cancellations. Previous military research on the topic of missile maintenance scheduling has indicated that this problem can be well defined as a facility location problem [2].

The terrorist acts of September 11, 2001 raised concerns as to whether mandated levels of security personnel used for maintenance dispatches were sufficient to protect all ICBMs from damage, destruction, or theft. These concerns resulted in major changes to Department of Defense and Air Force security instructions, which mandated the presence of additional security personnel during weapon system maintenance activities within the missile complex. Additionally, security changes decreased the security forces' response times to penetrated missile launch facilities. With a finite number of security personnel available each day, only a limited number of maintenance activities can be performed.
Missile maintenance schedulers must cope with these new security personnel constraints to maintain the highest missile alert rate possible, while still accomplishing the lower-priority tasks that are necessary to keep the missile infrastructure intact and support systems functioning.

This research seeks to answer the following question: how can ICBM maintenance scheduling methods be enhanced to compensate for given security requirements while sustaining prescribed readiness levels? Sensitivity analysis examines what effects manipulating various missile security requirements have on the scheduling of daily missile maintenance activities. This research addresses the operating environment as it existed during the time period that this study was performed, and is most concerned with security forces manpower limitations and security requirements that detrimentally impact the maintenance organization's ability to accomplish maintenance activities.

\section{Literature review}

\subsection{Previous military research}

Several attempts have been made to enhance the missile maintenance and security forces scheduling practices by the US Air Force. In 1999, Seaberg [3] proposed to geographically cluster all F. E. Warren AFB missile maintenance activities requiring launch facility penetration within designated security "security patrol areas," or patrol areas that are centered upon two of the 15 missile alert facilities. Dawson [2] developed a Microsoft Excel ${ }^{\circledR}$-based optimization tool that established security patrol area focal points at 233 locations within the missile complex vs. the normal 15 Minuteman III missile alert facilities proposed by Seaberg. Dawson's model produced results that closely paralleled Seaberg's [4] security patrol area concept, but offered security forces schedulers the option to place patrolling security teams based on several different objectives: maximizing the number of launch facilities covered by the security patrol area; minimizing total security team travel time; or minimizing longest travel time. This research focuses more on the maintenance scheduling aspect of missile maintenance and is the first effort of its kind.

\subsection{Location modeling}

One of the first covering models, the maximal covering location problem (MCLP) was first formulated by Church and ReVelle [5] in 1974. This model evolved 
from the location set covering problem (LSCP) [6]. However, unlike the LSCP, which covered all demand nodes within a maximum distance of a minimal number of facilities, the MCLP model considered that financial constraints could limit the number of facilities that would be placed. Since the development of the first MCLP, many variations of the original model have been constructed. The following examples of expanded MCLP models are by no means all inclusive, as numerous studies that have been performed.

In 1979, Church and Meadows "generalized the search for on optimal solution to a dominant set of points NIPS (Network Interest Point Set) which, according to Berman, includes in addition to the nodes all points that are $T$ units of distance away from any demand point" [7]. A two-level hierarchical covering location problem (HCLP) was developed in 1982 by Moore and ReVelle [8]. This particular problem looked at locating facilities that provided different levels of service to the demand nodes. Espejo et al. [9] expanded on the HCLP model, developing solutions using dual-based heuristics; specifically, a subgradientbased heuristic incorporating a Lagrangean-surrogate relaxation, which reduced to a $0-1$ knapsack problem. Megiddo et al. [10] considered locating new facilities within a pre-existing network of established facilities, with the goal of "drawing" a maximum number of customers. In addition, Berman [7] studied the relationship between $p$-maximal cover problems and partial center problems on networks. This study paved the way for the creation of the generalized maximal covering location problem (GMCLP), developed by Berman and Krass in 2002. Unlike the original MCLP, this model did not consider demand coverage as binary; instead, it assumed "that the coverage level is a decreasing step function of the distance to the closest facility..." [11]. ReVelle et al. considered an extension of the MCLP that not only required demand nodes within a certain distance to be covered by a facility, but also required that the facilities themselves be covered by other facilities within a different coverage radius. This redundancy of demand and facility coverage was applied to emergency services, where more than one facility may be required to cover overall network demand [12].

Additional studies closely parallel this specific research. Ma integrated scheduling and MCLP methodologies to determine police patrol areas for the Dallas Police Department. The objective of the Police Patrol Area Covering problem (PPAC) was to "maximize the number of incidents served or 'covered' within a desired response time" [13]. Also, the MCLP and set covering models have been applied to similar urban location problems $[14,15]$. Additional vehicle scheduling problems with locational aspects have been studied more recently [16-18], and the sensitivity of operating in a nuclear environment can be seen in [19-21]. In addition, problems for geographically dispersed services $[22,23]$ and for developing facility networks across large areas [24-27] are the focus of recent research. However, instances of scheduling for field service maintenance technicians over large geographic areas have only rarely been studied [28,29], and no known research uses location methods such as the MCLP for scheduling maintenance technicians over large geographic areas.

\section{Materials and methods}

In this research, maintenance scheduling activities at F. E. Warren AFB missile launch facilities are improved using the maximal covering facility problem (MCLP) methodology to ensure that a weighted sum of scheduled launch facility maintenance activities is maximized while meeting security constraints. Actual data collected from F. E. Warren AFB were used to reconstruct the 26 daily maintenance schedules for May 2005. These actual schedules served as a baseline of comparison to the research model outputs. Additionally, the problem is to assign jobs at different locations to a group of maintenance technicians with different job skills; similar to research by $\mathrm{Xu}$ [28].

\subsection{Modeling approach}

Based on examination of the various facility location methods found in the literature, the MCLP methodology was used as the basis for the research model. Microsoft Excel ${ }^{\circledR}$ with the Premium Solver ${ }^{\mathrm{TM}}$ plug-in was chosen as the optimization software to create the model, due to the availability and user-friendly nature of the software. The technique used to solve the location problem is highly dependent on several factors, which are specific to the individual problem. The type of objective function, decision variables, constraints, problem complexity, needs of the end user, and computational time required to solve the problem were all factors considered when developing this tool to produce problem solutions. Often in combinatorial optimization, heuristics are best to use when resources are constrained and they allow for optimal, or near optimal, solutions with reduced processing time. Over the course of developing a research model, it was found that a two-stage heuristic, each stage utilizing optimization techniques, was the most reasonable approach to produce the final model solutions for this problem. The existence of 
a complicated objective and the relatively large problem size made the choice of a two-stage model desirable. Extensive testing, the use of optimization techniques in both stages, and comparisons to field results give the researchers a high level of confidence that the solutions from the two-stage model are near optimal. Due to the heuristic approach, optimality cannot be guaranteed, but the approach achieves a major improvement over current scheduling techniques.

Specific Air Force guidance was used to develop the objective function of the research model. Air Force regulations [1] state that:

All maintenance actions and management efforts must be directed towards maximum availability of ICBMs in support of the United States Strategic Command requirements directives. All maintenance supervisors are mandated to use all resources in the most effective and efficient way with emphasis on the safety and welfare of the technician [1] .

To successfully fulfill this objective, maintenance efforts must focus on completing those maintenance activities that are crucial to keeping a maximum number of ICBMs on full alert status, given manpower, equipment, and environmental constraints. Regulations [1] provide further direction for which maintenance activities are most crucial to sustaining the "maximum availability of ICBMs," and provide a detailed breakdown of maintenance activity prioritization.

At the time of this study, US Air Force missile maintenance scheduling personnel built the daily schedule manually, one day prior to the day maintenance tasks were performed. A long-term maintenance forecast developed by schedulers dictates which life-extension programs, weapon system upgrades, and depot-level maintenance activities must be performed at the LFs. These programmed activities are combined with other outstanding maintenance work orders and assigned to the schedule according to the priority system dictated by regulation to form the daily schedule.

To quantify how effective the maintenance schedulers were at meeting the published objective with the 26 daily baseline schedules, a weighting factor was created for each type of maintenance activity. Due to the large variety of maintenance tasks that are performed, all tasks were grouped into 18 separate categories to create the weighting system for this research. Categories were based on the published priority designation [1], extent of coordination efforts required, mission impact, level of security presence required, and practicality of completion. A description of the categories is included in Appendix A. Weights were assigned to each category based on an exponential distribution of the 18 categories. An exponential distribution was chosen because of its ability to assign a greater weight and higher degree of separation to the maintenance tasks most critical to maximizing the quantity of ICBMs on alert, while gradually leveling the impact of the weights as the probability distribution frequency (PDF) curve approaches the least critical categories. After preliminary experimentation, a $\lambda$ value of 0.25 , multiplied by a factor of 1000 , produced category weights that provided a sufficient level of separation between high and low-priority categories, without making the weights of the last several categories equal. After the weights were applied to the maintenance activities in the 18 maintenance categories, a total weighted value of the maintenance required for each candidate launch facility was computed for each of the 26 daily baseline schedules. To fulfill the model objective and measure the overall scheduling effectiveness, the research model aims to maximize this weighted sum of maintenance activities at launch facilities included in the daily schedule.

\subsection{Calculations}

The problem of enhancing current missile maintenance scheduling processes is modeled as a two-stage MCLP.

\subsubsection{Model stage one formulations}

The notation used to define the model in stage one are as follows:

$L$, the set containing 150 launch facilities (A02, $\mathrm{A} 03, \ldots, \mathrm{O} 11)$;

$M$, the set containing 15 missile alert facilities (A01, B01, .., O01);

$P$, maximum number of missile alert facilities (security patrol area centers);

$R$, maximum security patrol area response time;

$i$, the index of candidate missile alert facilities;

$j$, the index of candidate launch facilities;

$w_{j}$, weight of maintenance activity at launch facility $j$;

$x_{j}$, binary condition indicating whether or not launch facility $j$ is covered;

$a_{i j}$, binary condition indicating whether or not the response time between missile alert facility $i$ and launch facility $j$ falls within maximum specified response time;

$r_{i j}$, the response time between missile alert facility $i$ and launch facility $j$;

$Y_{i}$, binary condition indicating whether or not missile alert facility $i$ is selected as the security patrol area center. 
The MCLP mathematical formulation for stage one is based on the original formulation developed by Church and ReVelle [5]:

$$
\begin{aligned}
& \operatorname{Maximize} \sum_{i=1}^{M} \sum_{j=1}^{L} Y_{i} w_{j} x_{j} \\
& \text { Subject to } \sum_{i \in M} a_{i j} Y_{i} \geqslant x_{j}, j=1, \ldots, L \\
& \sum Y_{i} \leqslant P \\
& Y_{i}=\{0,1\}, \forall i \\
& \text { where } a_{i j}=\left\{\begin{array}{ll}
1 & \text { if } r_{i j} \leqslant R \\
0 & \text { otherwise }
\end{array}, \quad \forall i \text { and } j\right. \\
& x_{j}=\left\{\begin{array}{l}
1 \text { if } \sum_{i \in M} a_{i j} Y_{i} \geqslant 1 \\
0 \text { otherwise }
\end{array}, j=1, \ldots L\right. \\
& Y_{i}=\left\{\begin{array}{ll}
1 & \text { if missile alert facility } i \\
& \text { is selected } \\
0 & \text { otherwise }
\end{array},\right. \\
& j \in L, i \in M
\end{aligned}
$$

The objective function (1) was based on Air Force regulations [1] and the requirement for the maximum availability of ICBMS. It maximizes the sum of weighted demands at covered launch facilities. Constraint (2) ensures that launch facility $j$ is not considered unless it falls within the maximum response time of at least one selected missile alert facility, $i$. Constraint (3) states that no more than $P$ missile alert facilities can be selected as a security patrol area center. Constraint (4) is the binary constraint placed on the decision variables. Condition (5) assigns $a_{i j}$ a value of 1 if the response time between missile alert facility $i$ and launch facility $j$ is less than, or equal to, the maximum allowable response time, $R$; a value of 0 is assigned if the maximum response time is exceeded. Condition (6) assigns $x_{j}$ a value of 1 if launch facility $j$ is covered by at least one selected missile alert facility, $i$, which is a member of the set of all missile alert facilities, $M$; a value of 0 is assigned if launch facility $i$ is not covered. Condition (7) assigns $Y_{i}$ a value of 1 if missile alert facility $i$ is chosen as the security patrol area center; otherwise, it takes on the value 0 . Condition (8) states that launch facilities, $j$, are members of the set of all launch facilities, $L$; likewise, missile alert facilities, $i$, are members of the set of all missile alert facilities, $M$.

\subsubsection{Model stage two formulations}

Stage two utilizes the solutions from stage one to compute the final model solution. The stage one solution is specifically passed to stage two through the determination of sets $X$ and $Y$. The notations used to define the model in stage two are as follows:

$X$, the solution set of launch facilities covered by the missile alert facilities selected in stage one;

$Y$, the solution set of missile alert facilities selected in stage one of the model;

$i$, the index of selected missile alert facilities;

$j$, the index of candidate launch facilities;

$t$, the index of maintenance/security team types (SETS, ..., TRN);

$w_{j}$, weight of maintenance activity at launch facility $j$;

$x_{j}$, binary condition indicating whether or not launch facility $j$ is selected;

$a_{i j}$, binary condition indicating whether or not the response time between missile alert facility $i$ and launch facility $j$ falls within the maximum specified response time;

$c_{j t}$, number of teams of type $t$ required at launch facility $j$;

$C_{t}$, total number of available maintenance/security teams, $t$.

The MCLP mathematical formulation for stage two is also based on the original formulation developed by Church and ReVelle [5]:

$$
\begin{aligned}
\text { Maximize } & \sum_{j=1}^{X} w_{j} x_{j} \\
\text { Subject to } & \sum_{i \in Y} a_{i j} \geqslant x_{j}, \quad j=1, \ldots, X \\
& \sum_{j=1}^{X} c_{j t} \leqslant C_{t}, \quad t=\text { SETS, } \ldots, \text { TRN } \\
& x_{j}=\{0,1\}, \forall j \\
\text { where } & x_{j}=\left\{\begin{array}{ll}
1 & \text { if launch facility } j \\
0 & \text { is selected } \\
0 & \text { otherwise }
\end{array},\right. \\
& j \in X, i \in Y
\end{aligned}
$$

The objective function (9) maximizes the sum of weighted demands at covered launch facilities. Constraint (10) ensures that launch facility $j$ is not selected unless it falls within the maximum response time of at least one of the two selected missile alert facilities, $i$. Constraint (11) states that the sum of maintenance and security teams $t$ required at all selected launch facilities must be less than, or equal to, the total number of each maintenance and security team available, $C_{t}$. Constraint (12) is the binary constraint placed on the decision variables. Condition (13) assigns $x_{j}$ a value of 1 if launch 


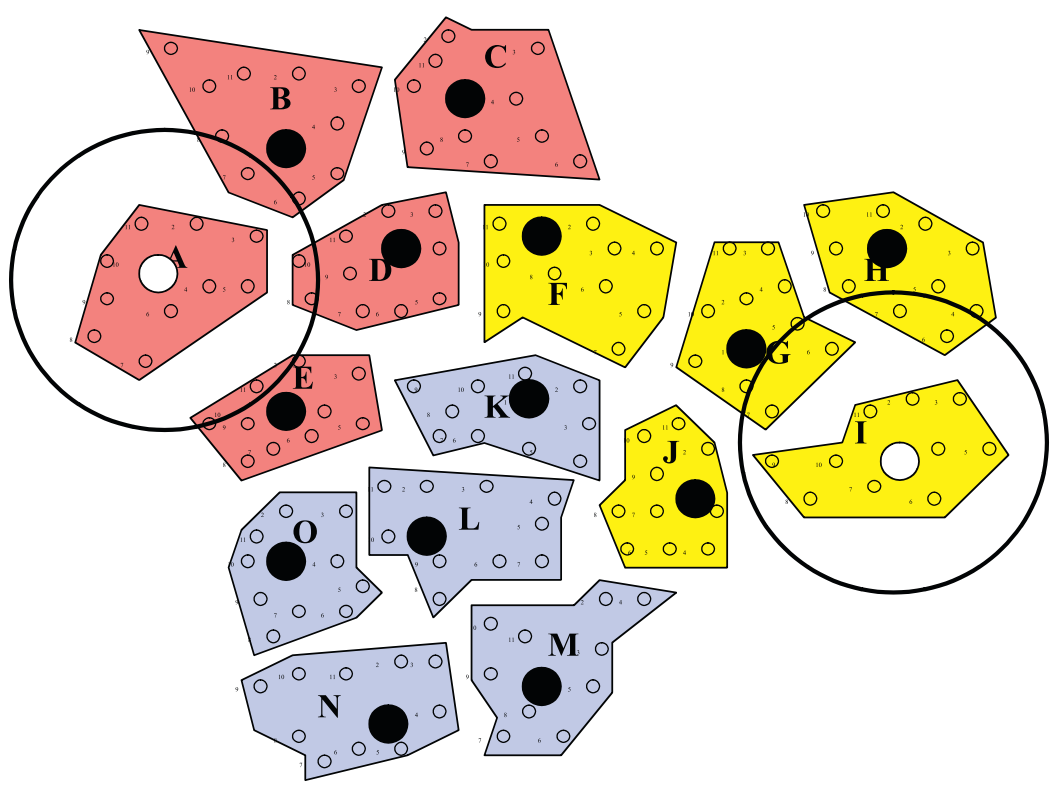

Fig. 1. Stage one solution set illustration.

facility $j$ is chosen for the schedule; otherwise, it takes on the value 0 . Condition (14) states that launch facilities $j$ are members of the solution set of launch facilities covered by the missile alert facilities selected in stage one, $X$; likewise, missile alert facilities $i$ are members of the solution set from stage one, $Y$.

\subsection{Generation of solutions}

To generate solutions for the original model, a twostage process was used.

\subsubsection{Model stage one solutions}

In stage one, the 15 missile alert facilities served as the decision variables, which were subject to the following three constraints: decision variables are binary; the solution set must include at least one, but no more than two missile alert facilities; and all launch facilities considered by the objective function must fall within at least one of the 60-min security patrol areas provided in the solution set. Fig. 1 illustrates what a stage one solution might look like.

The 15 large dots represent the missile alert facilities that can serve as staging area for security patrol teams. The 150 small dots represent the launch facilities within the missile complex. Assuming that all launch facilities have some sort of required maintenance activity to be performed, and given the previously mentioned constraints, stage one selects the two missile alert facilities, identified by a large white dot, that maximize the weighted sum of all maintenance demands at the 150 launch facilities. All launch facilities that fall within the security patrol areas of the stage one solution set, identified with the large black circles, make up the subset of launch facilities from which stage two of the model develops the final schedule. Note that the security patrol areas are for illustration purposes only and do not depict actual 60-min security response windows using geographical distance calculations.

\subsubsection{Model stage two solutions}

The model selected two missile alert facilities whose security patrol area(s) maximized the weighted sum of maintenance activities at the candidate launch facilities. The two missile alert facilities that made up the stage one solution set established the security patrol area focal points required to generate final solutions in stage two. Stage two maximized the weighted sum of the selected subset of launch facilities that fall within 60-min of the stage-one security patrol area(s), given maintenance and security personnel constraints. In other words, a subset of launch facilities covered by the stage one security patrol areas were picked to be on the daily schedule. Fig. 2 illustrates what a stage two solution might look like.

The stage-two solution provides a list of launch facilities covered by the security patrol areas that maximize the weighted sum of all required maintenance, given 


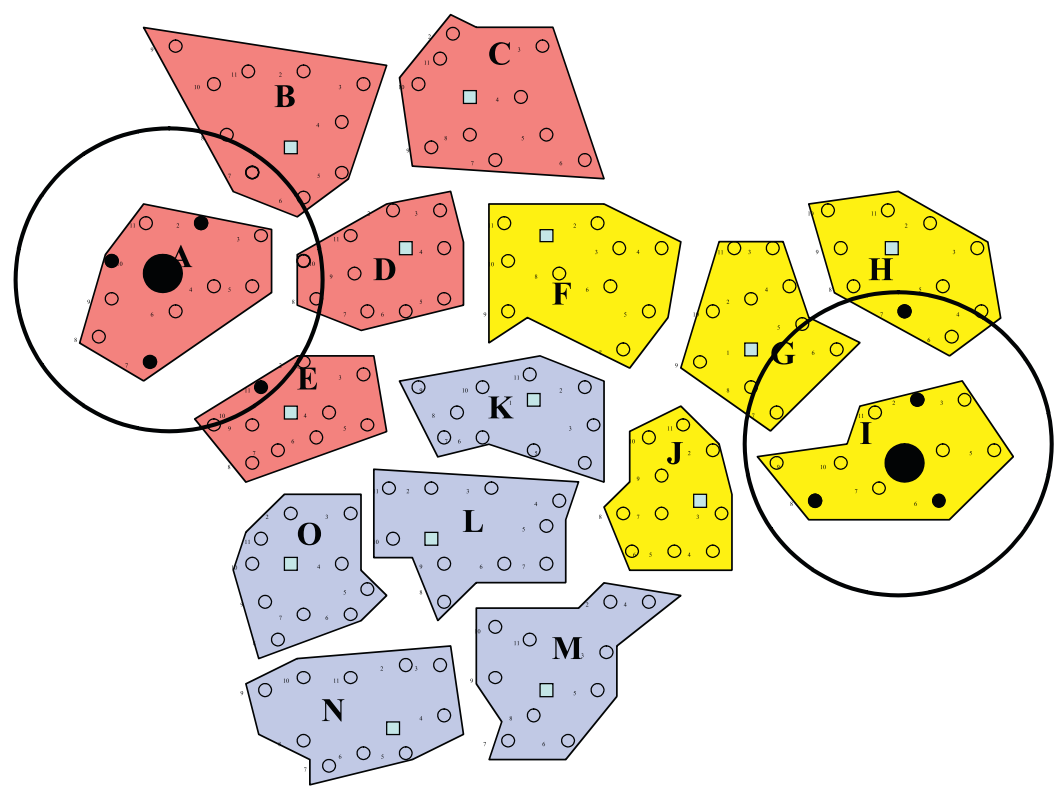

Fig. 2. Stage two solution set illustration.

the model constraints. The launch facilities that make up the model's final solution set are identified with the small, black dots. The maintenance teams and security personnel not utilized in the final model solution could then be assigned to maintenance activities exempt from security patrol areas, or removed from the daily schedule completely.

\section{Results}

\subsection{Model analysis}

The results generated using this research model were compared to the actual maintenance activities performed between May 1 and May 26, 2005 at F. E. Warren AFB, WY. Analysis was performed on the number of launch facilities chosen for the daily schedule, the weighted maintenance sum of launch facilities selected, and manpower utilization for both maintenance and security.

\subsubsection{Comparison of total launch facilities completed}

When comparing only the number of LFs selected, improvements in the schedule were realized by the research model in only 8 of the 26 daily schedules, with actual increases ranging from 0 to 3 additional launch facilities. In one instance, the actual baseline schedule completed one more launch facility than the research model; however, in this instance, the research model produced a higher weighted sum of maintenance than the actual schedule. In fact, a significantly greater weighted sum of maintenance was accomplished in most of the days where the same number of LFs was selected. Fig. 3 compares the historical number of daily launch facilities completed to the research model outputs.

\subsubsection{Weighted-sum solution comparisons}

In 18 of the 26 daily schedules analyzed, the research model produced better weighted-sum solutions than were provided by the actual schedule. Improvements ranged from 2 percent to nearly 35 percent. This represents a marked improvement over the current scheduling method, which does not utilize structured formulations. Fig. 4 compares the weighted maintenance sums of the actual schedule and the research model. Future model testing should also consider increasing the weight of maintenance tasks which are delayed in order to increase their priority.

\subsubsection{Manpower utilization rate comparisons}

Fig. 5 shows maintenance team utilization rates for the actual schedule and the research model schedule. Only small differences were noted in overall maintenance team utilization rates; the baseline schedule demonstrated an overall utilization rate of 71.48 percent, while the research model provided a 69.73 percent utilization rate. It was noted, however, that the 
Completed Launch Facilities

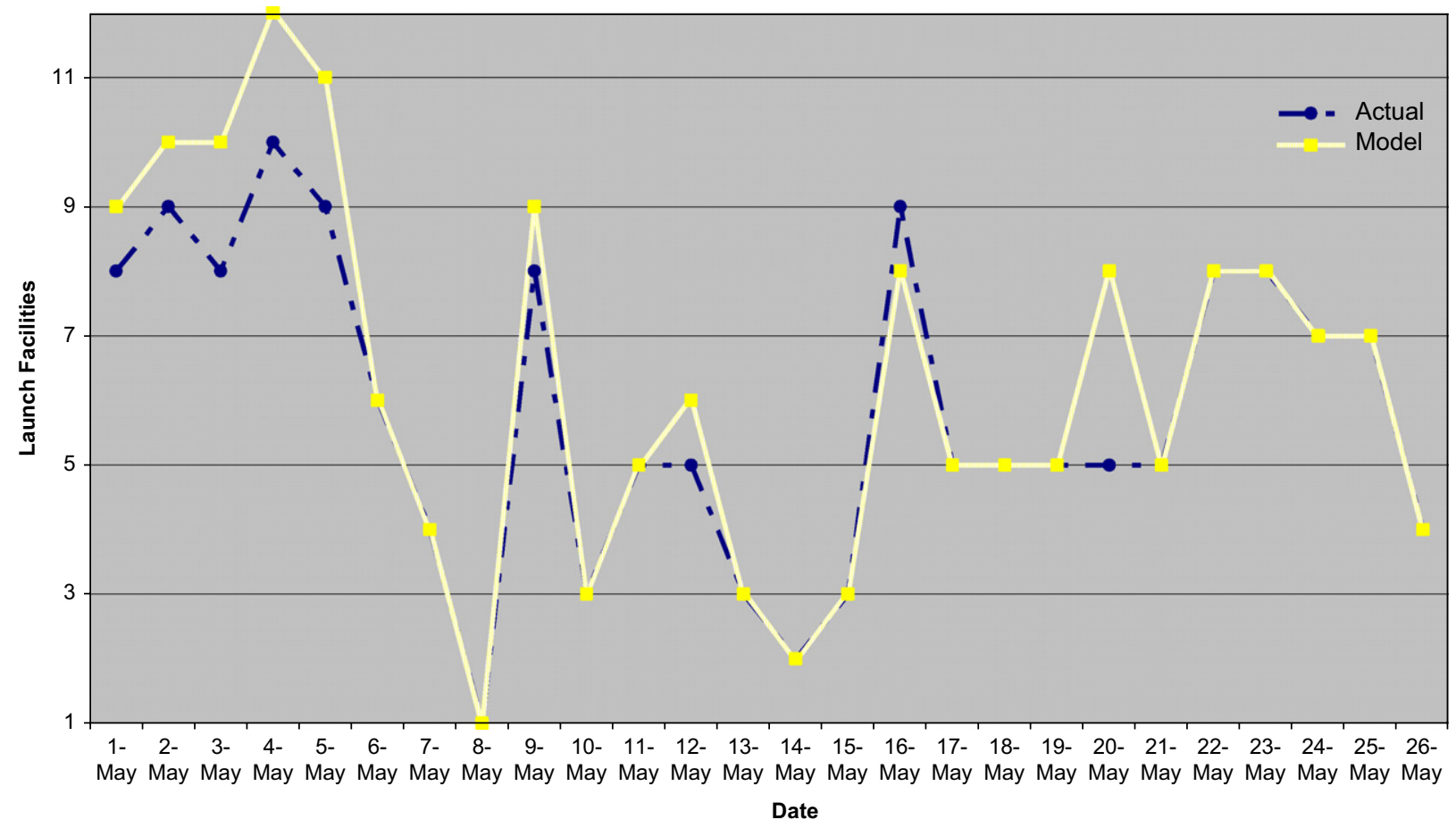

Fig. 3. Completed launch facilities: actual vs. model.

research model utilized more of the facility maintenance (FMT) and civilian periodic maintenance teams (CCT and RVM), while the baseline schedule utilized more electro-mechanical (EMT), training (TRN), and back shop maintenance teams (PNEU, PMT and MMT). The research model only showed slight improvements in security personnel utilization, with an overall 94.11 percent utilization, while the actual schedule demonstrated a 90.03 percent utilization rate.

\subsection{Sensitivity analysis}

Following initial analysis of the model outputs, each of the 26 maintenance schedules was run multiple times with different settings for the purposes of sensitivity analysis.

\subsubsection{Security response time sensitivity analysis}

The first sensitivity analysis model set relaxes the security force response time constraint, $R$, varying it from 60 to $20 \mathrm{~min}$, in 10 -min increments, to show the effects on the total weighted sum of maintenance activities, the number of launch facilities that are scheduled, and maintenance team/security personnel utilization. All other parameters remain unchanged in this analysis to isolate the effects of attenuated security patrol areas. From this analysis, missile maintenance and security forces managers can visualize how various response times will affect the total maintenance effort. Table 1 summarizes the impact of reduced response times on weighted sums of scheduled maintenance activities.

The response time radius and weighted sums are positively correlated; the weighted-sum totals increase as the security patrol area radius increases. By observing the weighted-sum values in Table 1 , the differences in weighted sums from the 60- to 30-min response times demonstrates that reducing the security patrol area size has minimal impact on the type and quantity of maintenance activities performed. However, reducing the security response times to $20 \mathrm{~min}$ appears to have a significant impact on the weighted sum of activities that can be performed, with average weighted sums decreasing by over 18 percent.

Only a slight decrease in average weighted sums is observed between the first four increments, with a larger total decrease of 84 points observed with the 20 -min response time. This large decrease at $20 \mathrm{~min}$ is equivalent to 1 high-priority maintenance activity or several lowpriority activities. The small decrease in weighted sums 
Research Model Weighted Sum Improvements

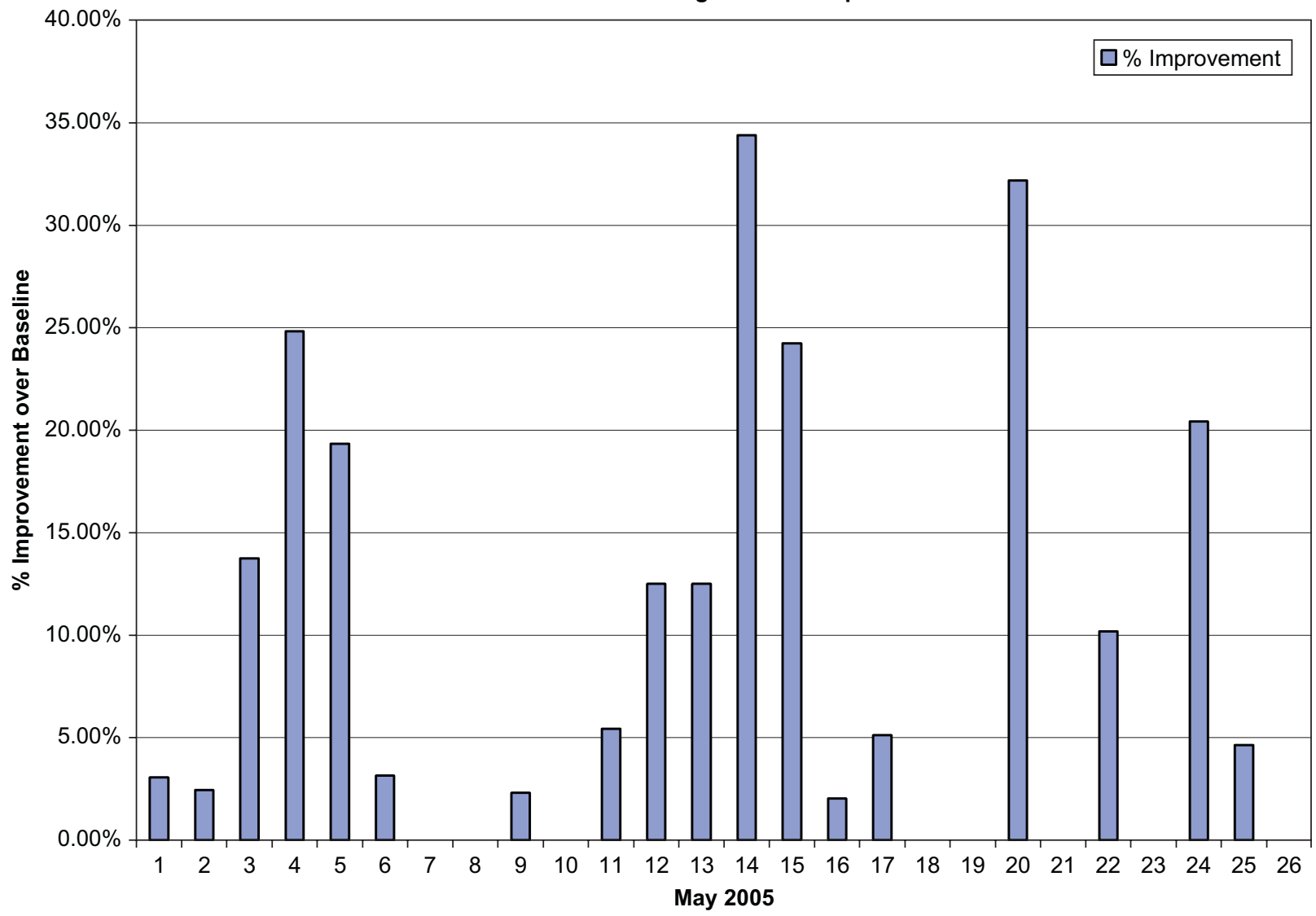

Fig. 4. Model improvements over actual schedule.

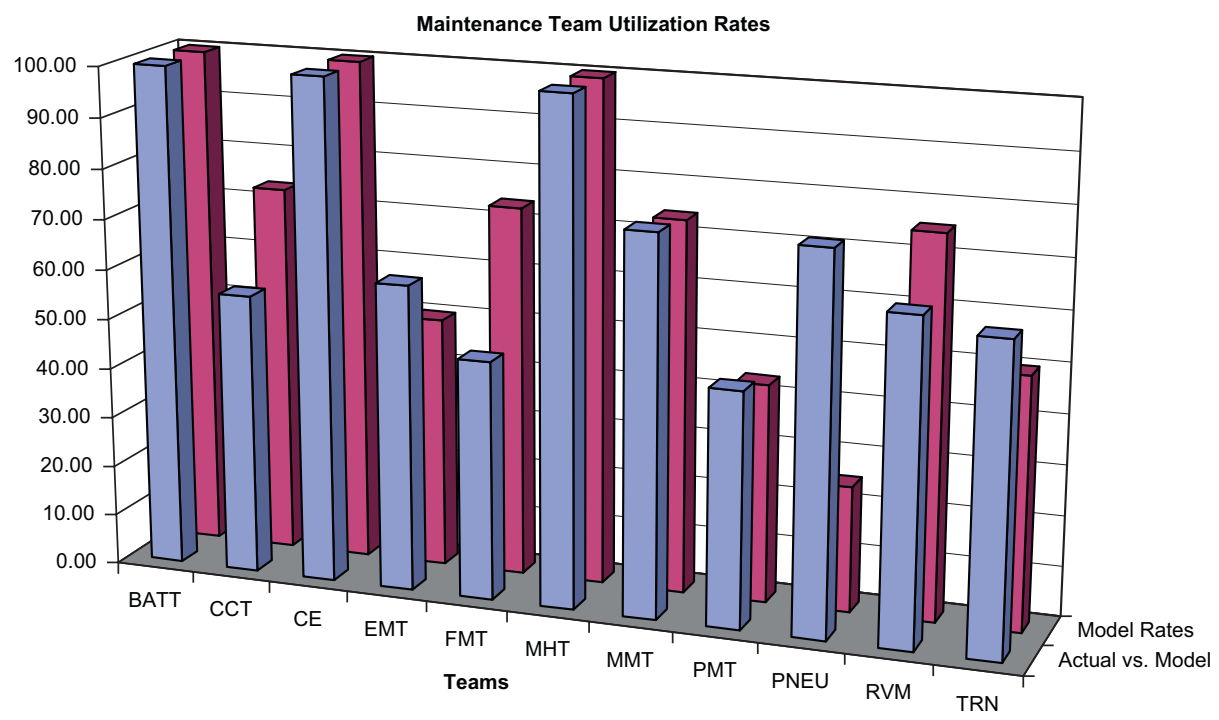

\begin{tabular}{|c|c|c|c|c|c|c|c|c|c|c|c|}
\hline & BATT & $\mathrm{CCT}$ & CE & EMT & FMT & MHT & MMT & PMT & PNEU & RVM & TRN \\
\hline$\square$ Actual vs. Model & 100.00 & 55.88 & 100.00 & 60.90 & 47.44 & 100.00 & 75.26 & 46.67 & 75.00 & 64.04 & 61.11 \\
\hline Q Model Rates & 100.00 & 73.53 & 100.00 & 50.00 & 73.72 & 100.00 & 73.95 & 43.33 & 25.00 & 75.44 & 50.00 \\
\hline
\end{tabular}

$\square$ Actual vs. Model $\square$ Model Rates

Fig. 5. Maintenance team utilization rates. 
Table 1

Weighted sums vs. response times .

\begin{tabular}{|c|c|c|c|c|c|}
\hline \multirow[t]{2}{*}{ May } & \multicolumn{5}{|c|}{ Weighted sum vs. response time } \\
\hline & 60 & 50 & 40 & 30 & 20 \\
\hline 1 & 540 & 506 & 497 & 497 & 460 \\
\hline 2 & 673 & 657 & 621 & 615 & 578 \\
\hline 3 & 753 & 753 & 651 & 553 & 434 \\
\hline 4 & 367 & 340 & 319 & 298 & 276 \\
\hline 5 & 358 & 306 & 306 & 306 & 269 \\
\hline 6 & 686 & 686 & 686 & 670 & 622 \\
\hline 7 & 75 & 75 & 75 & 75 & 43 \\
\hline 8 & 27 & 27 & 27 & 27 & 27 \\
\hline 9 & 1192 & 1181 & 1171 & 1021 & 942 \\
\hline 10 & 200 & 200 & 200 & 200 & 200 \\
\hline 11 & 224 & 213 & 224 & 224 & 171 \\
\hline 12 & 234 & 218 & 218 & 218 & 187 \\
\hline 13 & 99 & 99 & 99 & 88 & 99 \\
\hline 14 & 59 & 59 & 59 & 59 & 43 \\
\hline 15 & 82 & 82 & 82 & 82 & 77 \\
\hline 16 & 874 & 874 & 863 & 842 & 763 \\
\hline 17 & 248 & 248 & 248 & 242 & 216 \\
\hline 18 & 268 & 268 & 234 & 234 & 234 \\
\hline 19 & 1590 & 1590 & 1494 & 1494 & 1446 \\
\hline 20 & 220 & 220 & 220 & 186 & 186 \\
\hline 21 & 131 & 131 & 115 & 115 & 115 \\
\hline 22 & 238 & 238 & 206 & 195 & 174 \\
\hline 23 & 1325 & 1325 & 1309 & 1293 & 1251 \\
\hline 24 & 165 & 165 & 154 & 132 & 85 \\
\hline 25 & 495 & 495 & 495 & 458 & 400 \\
\hline 26 & 797 & 797 & 797 & 797 & 426 \\
\hline Average & 458 & 452 & 437 & 420 & 374 \\
\hline Avg decrease (\%) & & 1.40 & 4.61 & 8.38 & 18.42 \\
\hline
\end{tabular}

from the 60 -min security patrol areas to the $30-\mathrm{min}$ security patrol areas is attributable to the small number of maintenance activities available for the schedule, as well as the maintenance schedulers' abilities to tightly cluster scheduled maintenance activities. With the 20min security patrol areas, it is apparent that due to the geographic spacing of available jobs, the two security patrol areas are only able to cover the closest, highpriority maintenance activities. Extensive geographic clustering efforts would be required to cover more maintenance activities within these small security patrol areas.

Fig. 6 illustrates the impact of manipulating security response time constraints on the number of launch facilities scheduled daily.

The separation between the 60-, 50-, and 40-min response times appear drastic in the first few days, but then remain close through the remainder of the month. The 30-min response time also tracks with the previous three response times, but more significant decreases are observed throughout the graph. The 20-min response time obviously has the greatest impact, with large, steady reductions in scheduled launch facilities observed over the entire month of May. The 60-, 50-, and 40-min response times all averaged six scheduled launch facilities daily, while the 30- and 20-min response times averaged five and four scheduled launch facilities, respectively. Reducing the security response time can drastically impact an organization's ability to perform maintenance. If maintenance activities within the daily schedule are not geographically clustered, it will be difficult to maximize maintenance efforts and improve manpower utilization rates. Fig. 7 examines the impact of reduced response times on personnel usage.

Reducing the response time from 60 to $30 \mathrm{~min}$ appears to have a limited effect on personnel usage. However, the effects of the 20-min response time had more drastic impacts on total maintenance team utilization, with reductions ranging from 0 percent for the Battery teams, to nearly 53 percent for corrosion control. The overall average utilization rate decreased by 25.6 percent for maintenance teams, while security forces personnel averaged a 14.57 percent reduction. In summary, as the security patrol area response radius decreases, daily schedules tend to utilize more maintenance teams that work on the higher-priority maintenance activities.

\subsubsection{Impacts of adjusting security patrol area constraints}

The second sensitivity analysis examines how adjusting the security patrol area quantity constraint affects the weighted sums of daily scheduled maintenance, as well as the number of scheduled launch facilities. The number of permitted security patrol areas within the model is setup as a "less than, or equal to," constraint, with the maximum allowable security patrol areas establishing the upper bound. The security patrol area quantity constraint was varied between 1 and 5 , in increments of 1, while solutions for all 26 daily schedules were computed at each increment. For each security patrol area quantity, the minimum response time required for each daily schedule to reach the best-achieved model solution was recorded. Fig. 8 provides a breakdown of the minimum response time required to achieve the optimal weighted-sum solution for each daily schedule.

The first row of the table illustrates that with the model security patrol area constraint set to five, 14 of the 26 daily schedules could achieve the best solution with a 30-min response time, while the remaining 12 daily schedules only required a 20 -min response time to reach the best-achieved solution. In essence, 
Scheduled Launch Facilities vs. Response Time
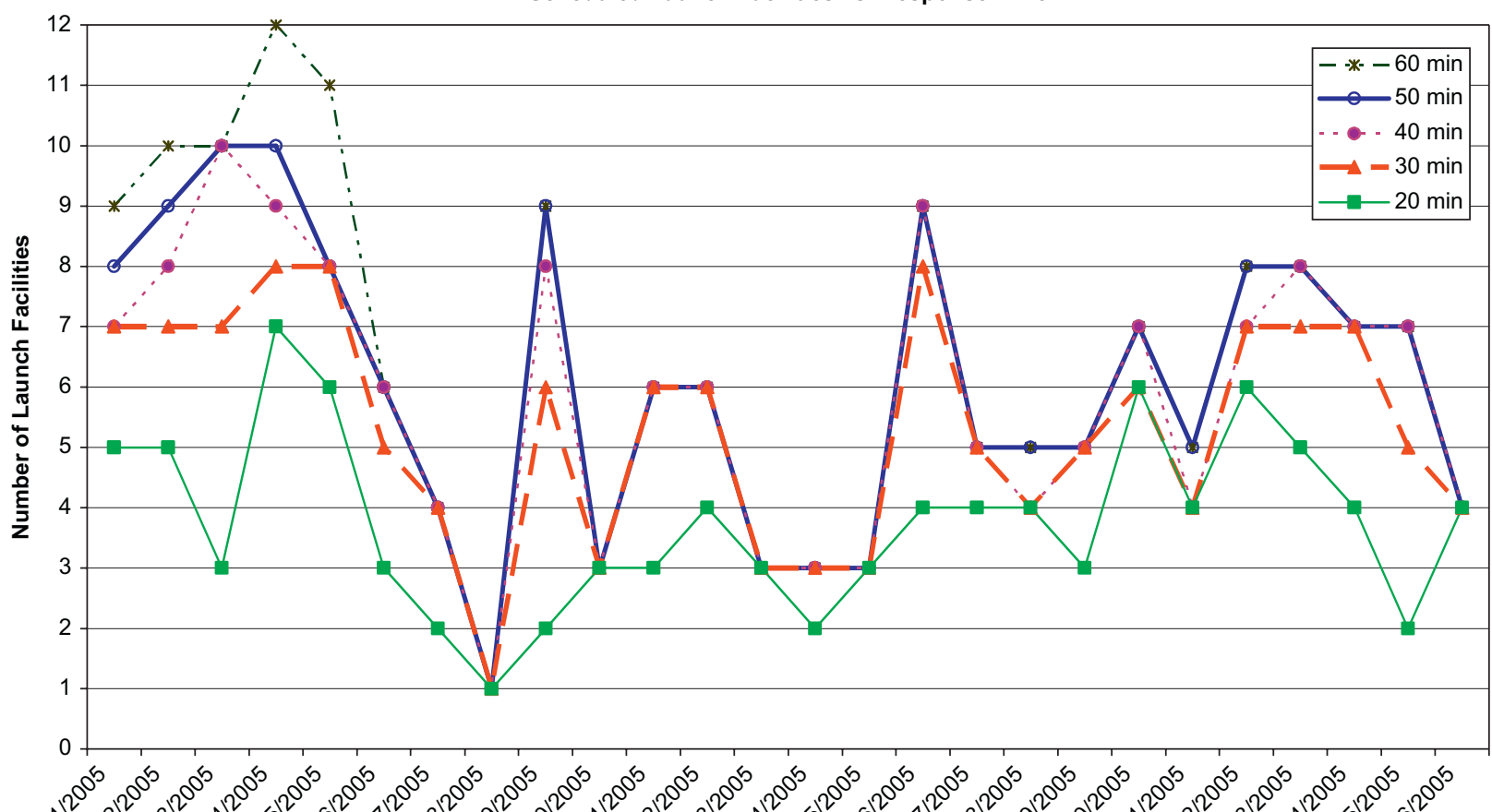

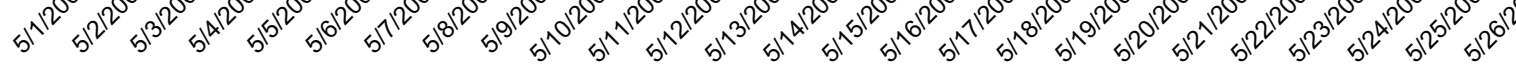

Date

Fig. 6. Response times vs. total scheduled launch facilities.

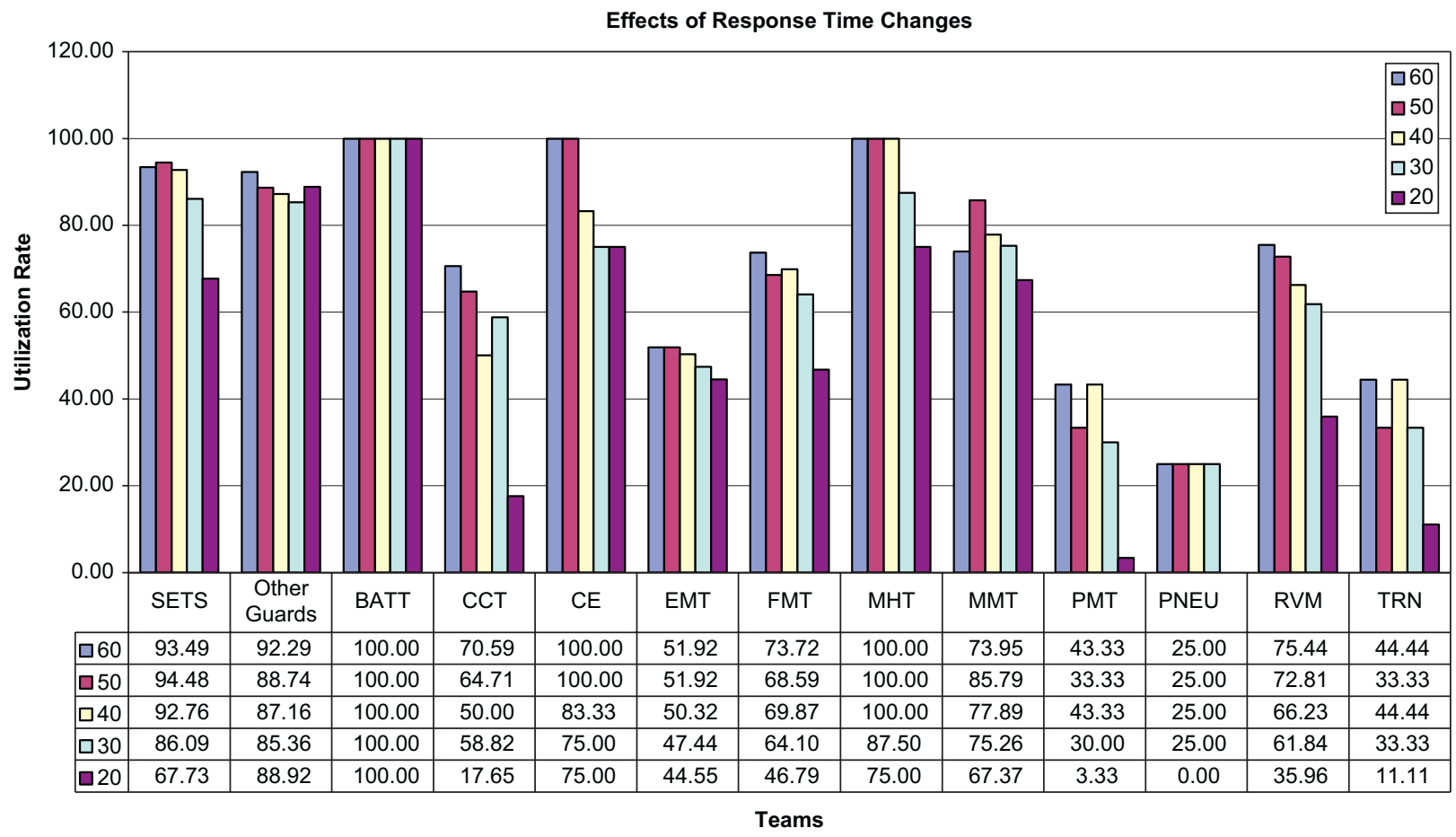

Fig. 7. Impact of reduced response times on personnel utilization. 


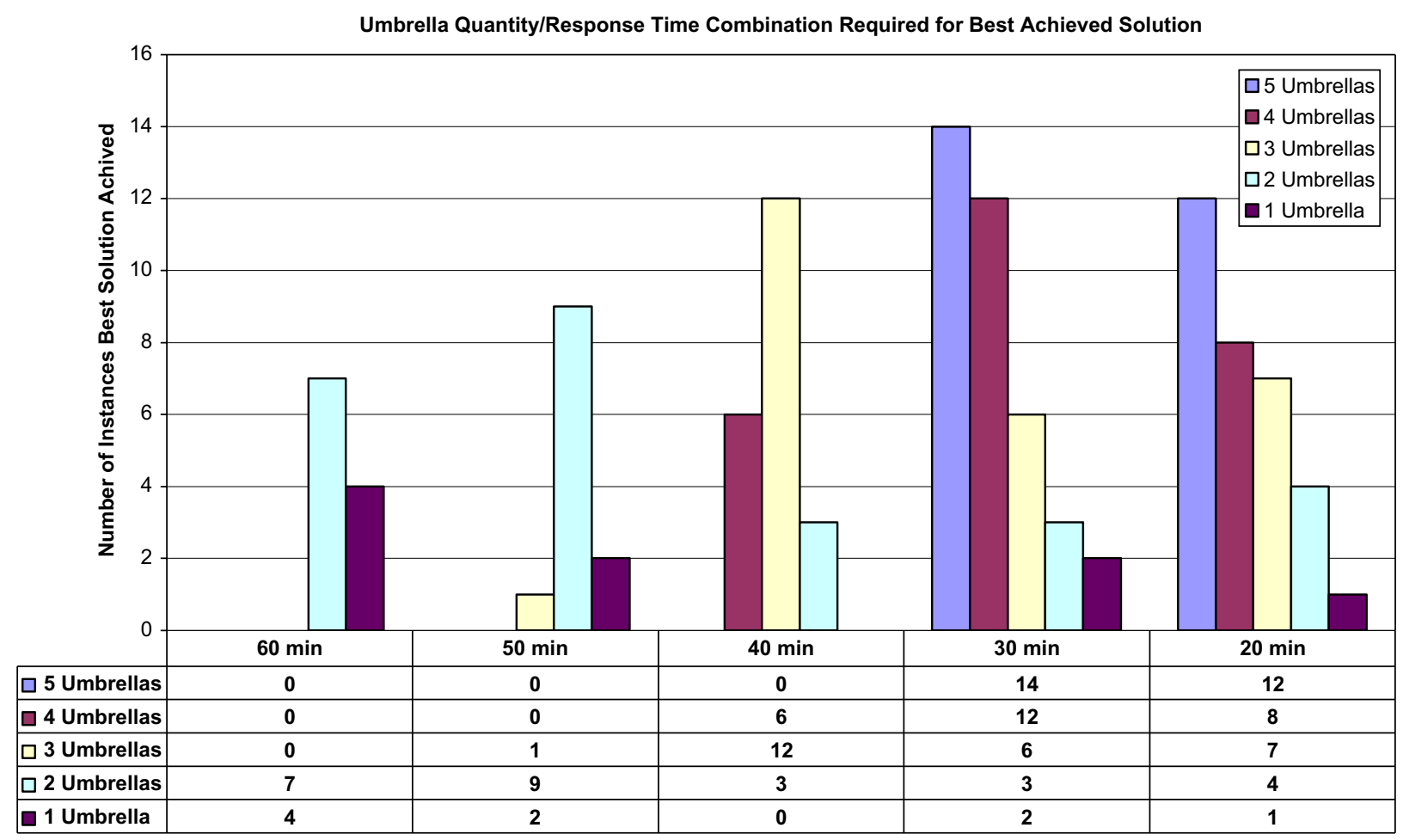

Number of Instances (out of 26 schedules)

Fig. 8. Minimum response time required to reach best-achieved solution.

with five security patrol areas in place, all 26 daily schedules could achieve near-optimal solutions with a 30-min response time or less. As the security patrol area constraint tightens, it appears that a negative correlation exists between the number of security patrol areas utilized and the minimum response time required to achieve the best achieved solution. As the quantity of security patrol areas decreases from five to one, the minimum response times required for all 26 daily schedules to achieve the best solution increases from 30 to $60 \mathrm{~min}$. With the model constraints set to one security patrol area, only nine of the 26 daily schedules could reach the best-achieved model solution; all other schedule solutions were less than optimal. In one instance, optimality could be achieved with one security patrol area and a 20-min response time. This is because only one launch facility was scheduled for that particular day and it was in close proximity of the security patrol area center. In two instances, optimality was achieved at $30 \mathrm{~min}$ and one security patrol area. For these particular dates, four or less launch facilities were tightly clustered within $30 \mathrm{~min}$ of the missile alert facility serving as the security patrol area center. However, these were isolated occurrences, as only nine of the 26 schedules could even achieve optimality with one security patrol area established.

During analysis, it was found that in the majority of cases involving four or five security patrol areas, the model did not fully utilize the number of security patrol areas allotted. This was especially true when 50- and 60-min response times were established. As such, sensitivity analysis demonstrates that utilizing more than three security patrol areas centered upon missile alert facilities would be wasting the additional security personnel required to support the unneeded security patrol areas. However, as shown in Dawson [2], if other locations in addition to missile alert facilities were considered, more than three security patrol areas could be better utilized. In conclusion, it is evident that in all 26 cases, a combination of 60-min response radius and two established security patrol areas are sufficient to maximize the weighted sum of available daily maintenance activities. This parallels with the security patrol areas concept of operations that were employed at F. E. Warren AFB during this study. However, utilizing the third security patrol area would allow for a 50-min response radius and still maximize the weighted sum of maintenance activities performed. 


\section{Conclusions}

Research analysis indicates that the two-stage research model does provide solutions that are as good, or better, than actual schedules produced during May 1-26, 2005 at F. E. Warren AFB. This research has demonstrated that the manual scheduling methods being used at F. E. Warren AFB can be enhanced through the use of optimization techniques. The time required to develop daily schedules can be reduced, while the energy invested in making schedule changes can be alleviated. The research model is able to select the two security patrol areas that best utilize all available maintenance teams and security personnel, given the resource and security constraints. From this set of missile alert facilities, the model is then able to produce feasible solutions that geographically cluster maintenance activities and maximize the weighted sum of maintenance activities performed. In summary, the model produced in this research can be an effective tool for maintenance schedulers to supplement current scheduling processes.

The number of launch facilities selected by the research model was highly dependent on the random geographic clustering of required maintenance activities. Daily schedules that had more tightly clustered, higherpriority maintenance activities available, generally had more launch facilities selected by the model. As was observed with the weighted-sums analysis, the weights assigned to maintenance categories greatly impacted the type and number of launch facilities scheduled. Reducing the weights assigned to the higher-priority maintenance activities could increase the total number of launch facilities scheduled, but at the expense of having several tightly clustered, low-priority maintenance activities bumping an important maintenance requirement, such as an off-alert launch facility, from the final schedule.

The first sensitivity analysis monitored the effects of decreased security response times on the weighted sum of maintenance activities scheduled, number of launch facilities scheduled, and manpower utilization rates. As was expected, smaller security patrol areas resulted in a decrease in the final weighted-sum value of maintenance performed. The weighted sum decrease was less profound in the 30-60-min security patrol area models, but more severe in the 20-min model. The small weightedsum differences observed between the 30-min and the 60-min models is attributable to the large weights assigned to the high-priority maintenance categories. As security patrol area response times decreased, security patrol area centers shifted to the missile alert facilities that covered those areas containing the high-priority maintenance activities. At each 10-min decrement, maintenance activities with small weights, such as periodic maintenance and training, were gradually removed from the schedule. With the 20-min security patrol area model, only the highest-priority launch facilities were selected.

In the second round of sensitivity analysis, the number of available security patrol areas was adjusted between one and five. As with the first sensitivity analysis, response times were also adjusted between 20 and $60 \mathrm{~min}$ in 10-min increments. It was found that as the number of available security patrol areas increased, the best achieved weighted-sum solution could be realized with a shorter response time. Final results suggested that two, 60-min security patrol areas were sufficient to maximize the weighted-sum solution for all 26 schedules, given current security and maintenance team availability. Additional security patrol areas could be created if security forces personnel were to abandon the deployment philosophy that assigns more than one fire team per security patrol area. Otherwise, major increases in security forces manpower would be required to create additional security patrol areas.

For the US Air Force, this model has provided the basic formulations and framework from which expand into an all-encompassing user-friendly scheduling solution. The techniques discussed in this paper have been presented to Air Force Space Command and 20th Air Force Logistics Divisions for potential application in future missile maintenance scheduling software development and acquisition. In addition, this research is believed to fill an important niche in the literature as the first application of location analysis techniques to a geographically dispersed scheduling problem for field service (maintenance) technicians.

\section{Disclaimer}

The views expressed are those of the authors and do not represent the official policy or positions of the United States Air Force, Department of Defence, or the U.S. Government.

\section{Appendix A. Explanation of 18 weighted mainte- nance categories}

1. Limited life component/reentry system (LLC/RS): Requires an enormous amount of coordination between organizations; requires additional security and has considerable mission impact.

2. Priority maintenance letter (PML) off-alert: Critical to accomplishing the published mission, often 
requires additional security, and entails a large amount of coordination.

3. Off-alert: Critical to accomplishing the published objective, often requires additional security personnel, and entails a large amount of coordination.

4. Priority 1: Usually does not require the amount of coordination and security that the previous categories require.

5. Concrete headworks: Accomplishment of these programs are critical to achieve security requirements outlined in instruction DoD S-5210.41-M; will directly impact ability to accomplish missile maintenance activities in the near future.

6. Propulsion replacement program (PRP): Includes maintenance activities that do not fall into previous category; requires some coordination, but not as much security.

7. Non-mission capable missile alert facility (NMC $M A F)$ : Priority varies depending on the number of other MAFs available; security may be required, depending on task.

8. Launch facility security check out (LF Security $C / O)$ : Requires additional security to maintain LF control until discrepancy is eliminated; can impact the number of security force personnel available to maintenance teams in successive daily schedules.

9. Time sensitive (TS) priority 3: Security escort requirements can vary, depending on level of LF access required.

10. Corrosion control (CCT): Periodic corrosion maintenance activities; number of security personnel required is dependent on LF access and components.

11. Periodic maintenance team (PMT): Periodic maintenance activities; number of security personnel required is dependent on level of LF access and components.

12. Rivet mile $(R V M)$ : Lower priority periodic maintenance activities; number of security personnel required is dependent on level of LF access and components.

13. PRP open hole: Periodic maintenance activities; requires coordination with some organizations to begin; limited timeframe for completion without further coordination.

14. Priority 2-3: Maintenance activities that do not fall into previous categories; may require presence of security depending on level of LF access.

15. Batteries: Utilizes special electro-mechanical team (EMT) to complete; requires security presence.

16. Training: Can be used to completemission essential tasks.
17. Priority 4-7: Maintenance tasks that do not fall into previous categories; usually not practical to complete when security is required; often completed in conjunction with higher priority maintenance activities; minimal impact to published mission.

18. Miscellaneous missile alert facility (Misc. MAF): Low-priority tasks that generally do not require presence of security personnel; any tasks at a MAF not falling within a higher category; generally have little to no direct impact on the published mission.

\section{References}

[1] Air Force Space Command. Intercontinental Ballistic Missile Maintenance Management, Air Force Space Command Instruction 21-114, Colorado Springs, CO; 1 May 2003.

[2] Dawson MC, Bell JE, Weir JD. A hybrid location method for missile security team positioning. Journal of Business and Management 2007;13:5-19.

[3] Seaberg JJ. Security patrol area. In: Presentation to 90th Space Wing, F. E. Warren AFB, WY; May 1999.

[4] Seaberg JJ. Proposed concept of operations for security patrol area security and clustering of maintenance operations. In: Proposal to 20th Air Force, F. E. Warren AFB WY; January 2005.

[5] Church RL, ReVelle CS. The maximal covering location problem. Papers of the Regional Science Association 1974;32:101-18.

[6] Toregas C, Swain R, ReVelle CS. The location of emergency service facilities. Operations Research 1972;19:1363-73.

[7] Berman O. The $\mathrm{p}$ maximal cover $-\mathrm{p}$ partial center problem on networks. European Journal of Operational Research 1994;72:432-42.

[8] Moore GC, ReVelle CS. The hierarchical service location problem. Management Science 1982;28:775-80.

[9] Espejo LGA, Galvao RD, Boffey B. Dual-based heuristics for a hierarchical covering location problem. Computers \& Operations Research 2003;30:165-80.

[10] Megiddo N, Zemel E, Hakimi SL. The maximum coverage location problem. SIAM Journal on Algebraic Discrete Methods 1983;4(2):253-61.

[11] Berman O, Krass D. The generalized maximal covering location problem. Computers Operations Research 2002;29:563-81.

[12] ReVelle CS, Schweitzer J, Snyder S. The maximal conditional covering problem. Information Systems and Operational Research 1996;34(2):77-91.

[13] Ma L. Integrating GIS and combinatorial optimization to determine police patrol areas. Unpublished Masters thesis, University of Texas at Dallas, Dallas, TX; 2003.

[14] Adenso-Diaz B, Rodriguez F. A simple search heuristic for the MLCP: application to the location of ambulance bases in a rural region. Omega 1997;25(2):181-6.

[15] Bautista J, Pereira J. Modeling the problem of locating collection areas for urban waste management. An application to the metropolitan area of Barcelona. Omega 2006;34(6): $617-29$.

[16] Day JM, Wright PD, Schoenherr T, Venkataramanan M, Gaudette K. Improving routing and scheduling decisions at a distributor of industrial gasses. Omega 2009;37(1):227-37. 
[17] Yan S, Tang C-H. Intercity bus scheduling under variable market share and uncertain demands. Omega 2009;37(1):178-92.

[18] Li J-Q, Borenstein D, Mirchandani PB. Truck scheduling for solid waste collection in the City of Porto Alegre, Brazil. Omega 2008;36(6):1133-49.

[19] Geldermann J, Bertsch V, Treitz M, French S, Papamichail KN, Hamalainen RP. Multi-criteria decision support and evaluation of strategies for nuclear remediation management, Omega 2009;37(1):238-51.

[20] Govindaraj T. Characterizing performance in socio-technical systems: a modeling framework in the domain of nuclear power. Omega 2008;36(1):10-21.

[21] Chen Y-W, Wang C-H, Sain J-L. A multi-objective geographic information system for route selection of nuclear waste transport. Omega 2008;36(3):363-72.

[22] Harper PR, Shahani AK, Gallagher JE, Bowie C. Planning health services with explicit geographical considerations: a stochastic location-allocation approach. Omega 2005;33(2): 141-52.
[23] Fugerholt K, Lindstad H. Optimal policies for maintaining a supply service in the Norwegian Sea. Omega 2000;28(3): 269-75.

[24] Fleischmann B, Paraschis JN. Solving a large scale districting problem: a case report. Computers Operations Research 1988;15(6):521-33.

[25] ReVelle C, Murray AT, Serra D. Location models for ceding market share and shrinking service. Omega 2007;35(5):533-40.

[26] Muppani VR, Adil GK. Efficient formation of storage classes for warehouse storage location assignment: a simulated annealing approach. Omega 2008;36(4):609-18.

[27] Srivastava SK. Network design for reverse logistics. Omega 2008;36(4):535-48.

[28] Xu J, Chiu SY. Effective heuristic procedures for a field technician scheduling problem. Journal of Heuristics 2001;7(5):495-509.

[29] Blumburg DF. Strategies for improving field serve operations productivity and quality. The Services Industries Journal 1994;14(2):262-77. 